\title{
Population-based survival for malignant mesothelioma after introduction of novel chemotherapy
}

\author{
Ronald A.M. Damhuis*, Caroline Schroten* and Jacobus A. Burgers\#
}

ABSTRACT: Malignant mesothelioma is known for its dismal prognosis and poor response to conventional treatment. Chemotherapy with cisplatin-antifolate combinations recently showed promising response rates and prolonged survival in randomised trials.

To assess the impact of this development on clinical practice and survival at a population-based level, treatment patterns and survival trends were studied for patients diagnosed with mesothelioma in the period 1995-2006. 4,731 records were retrieved from the Netherlands Cancer Registry and chemotherapy use and median survival were analysed.

For the periods $1995-1998$ to $2005-2006$, chemotherapy use increased from $8 \%$ to $36 \%$. Median survival increased over time from 7.1 months to 9.2 months. For pleural mesothelioma, multivariable analysis demonstrated that survival was poorer for elderly patients and sarcomatoid tumours. The prognostic impact of chemotherapy increased with time. Median survival for chemotherapy treated patients improved from 10.1 months (1995-1998) to 13.1 months (2005-2006). For peritoneal mesothelioma, median survival was poor (3.9 months) but better for females and younger patients.

This study demonstrates that chemotherapy use increased at a national level and coincided with an improvement in survival. The novel chemotherapy regimen appears to be more effective but, due to the observational nature of this study, alternative explanations cannot be excluded.

KEYWORDS: Antifolate, cancer registry, elderly, patterns of care, peritoneum, pleura

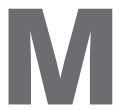
alignant mesothelioma (MM) is an aggressive disease, known for its relationship with asbestos exposure and its poor prognosis. Long-term survival is rarely seen and local treatment with radiotherapy or surgery tends to be unsuccessful. Response to single agent or combination chemotherapy used to be limited [1] until a novel agent was introduced, pemetrexed, that showed a response rate of $32 \%$ in combination with carboplatin [2]. Randomised trials subsequently showed a survival benefit of 10 weeks for combination treatment with pemetrexed-cisplatin [3] or raltitrexed-cisplatin [4], compared with singleagent treatment with cisplatin.

At a population level, the impact of new treatment strategies is often difficult to predict or determine. Trials tend to be restricted to young patients with limited disease, whereas most patients are diagnosed after age $65 \mathrm{yrs}$, when comorbidity or performance status may preclude cytotoxic treatment [5]. Moreover, availability of new drugs may be restricted due to the costs and some drugs, like raltitrexed, may not even become registered for use in clinical practice.
To assess the application of chemotherapy treatment over time and its impact on survival in a population-based setting, we analysed patient records from the Netherlands National Cancer Registry (NNCR) for the period 1995-2006.

\section{METHODS}

In $57 \%$ of cases, mesothelioma type was specified. Epithelioid tumours were most common $(46 \%)$ against $5 \%$ for sarcomatoid tumours and $6 \%$ with mixed tumour type. Incidence increased in time from an average annual number of 344 in the period 1995-1998 to an average number of 486 in 2005-2006.

Electronic records for patients with MM, diagnosed from 1995 through 2006, were derived from the NNCR. This registry covers a nation with 16.6 million inhabitants and approximately 100 hospitals. Newly diagnosed cancer patients are notified to the registry through hospital discharge diagnoses and notes from pathology departments. Due to the high incidence rates in the Netherlands, most pathologists have considerable experience with the differential diagnosis

\section{AFFILIATIONS}

${ }^{*}$ Rotterdam Cancer Registry, Rotterdam, and \#Dept of Thoracic Oncology, The Netherlands Cancer Institute-Antoni van Leeuwenhoek Hospital, Amsterdam, The Netherlands.

\section{CORRESPONDENCE}

R.A. Damhuis

Rotterdam Cancer Registry

P.0. Box 289

3000 AG Rotterdam

The Netherlands

E-mail: R.Damhuis@iknl.nl

Received:

Sept 062011

Accepted after revision:

Nov 072011

First published online:

Dec 012011 
of mesothelioma but still $60 \%$ of cases are sent for review to the national mesothelioma panel, mainly to substantiate compensation requests. After notification, trained registration clerks collect data from the clinical records, including sex and age, tumour site and morphology, and date of diagnosis. Date of diagnosis is defined as the day of acquiring cytology or histology material for microscopical verification. Annual follow-up information is obtained from the Centralized Civil Register.

Mesothelioma of the pericardium and tunica vaginalis testis were excluded, as were all cases in which diagnosis was based on clinical examination only. As of 2003, the TNM stage has been recorded by the NNCR, but this information was not used because of the absence of a standard staging policy. Stage information based on radiological examinations is considered unreliable and surgical staging is only performed in a minority of cases. Only the initial treatment was recorded, and noted as surgery, radiotherapy and/or chemotherapy. Information on treatment after progression was not recorded. As the surgery code may include diagnostic surgery, and radiotherapy may have been applied to prevent tumour invasion into biopsy or drainage intervention sites, both information on surgery $(3.7 \%$ of cases) and radiotherapy (13\% of cases) was ignored in the analyses. Curative surgery as part of multimodality treatment is mainly used in experimental studies and has only been performed in a limited number of highly selected patients [6]. Unfortunately, chemotherapy regimens are not coded in a standard manner by the NNCR. Information on the type of chemotherapy used was available only for the Rotterdam Registry subset, $\sim 20 \%$ of all cases. The year of diagnosis was recoded into four periods from 1995-1998, 1999-2002, 20032004 and 2005-2006. The international expanded access programme for pemetrexed [7] included the Netherlands from 2004 and comprised 181 patients from 12 centres [8]; this also included referrals from other hospitals. Pemetrexed became registered at the end of 2005 .

Chemotherapy use was tabulated for categorical subgroups and tested for significance with Chi-squared analysis and multivariable logistic regression. Results for significant $(p<0.05)$ predictors are reported with odds ratios and 95\% confidence intervals. Survival was calculated from the date of diagnosis until date of death or censored at January 1, 2009, using actuarial analysis. Survival patterns for pleural and peritoneal mesothelioma proved nonproportional. Therefore, multivariable regression using proportional hazards analysis was performed separately for both sites. Prognostic factors were tested for significance using the likelihood ratio test and were included in the model for $p<0.05$. For pleural mesothelioma, a chemotherapy-period interaction term was introduced to account for the time-dependent effect of chemotherapy.

\section{RESULTS}

This study comprised a total of 4,731 patients with MM, 4,115 (87\%) males and 616 (13\%) females (table 1). Median age was 68 yrs, for both pleural and peritoneal mesothelioma, and 35\% of patients were aged $70-79 \mathrm{yrs} ; 11 \%$ were aged $\geqslant 80 \mathrm{yrs}$. Peritoneal mesothelioma was uncommon (5.6\%) and more frequent in females $(10.7 \%)$ than in males $(4.9 \%)$.

Chemotherapy use increased in time, from $8 \%$ in the period 1995-1998 to 36\% in the period 2005-2006 (OR 8.4) (table 1).
Chemotherapy was used less often in the elderly; from $11 \%$ for those aged $70-79 \mathrm{yrs}$ (OR 0.22 ) to $3 \%$ in patients aged $\geqslant 80 \mathrm{yrs}$ (OR 0.04). Patients with epithelioid tumours were more often treated with chemotherapy $(22 \%)$ than those with sarcomatoid tumours $(15 \%)$ or mixed type tumours $(15 \%)$. Sex and tumour site had no significant impact on chemotherapy use. The novel antifolate-platinum schedule was introduced in 2003-2004 and became the standard regimen in 2005-2006 (table 2).

Median survival was 8.1 months for pleural mesothelioma and 3.9 months for peritoneal mesothelioma (table 3). Median survival for patients with sarcomatoid tumours was significantly worse (3.6 months) than for patients with mixed type tumours (7.1 months) or those with epithelioid tumours (10.1 months). Elderly patients had half the survival time (5.2 months for those aged $\geqslant 80 \mathrm{yrs}$ ) of younger patients ( 10.5 months for those aged 20-59 yrs). For patients who received chemotherapy, median survival improved with time from 10.1 months in the first period to 13.3 months in the last period. 2-yr survival was only $13 \%$.

For pleural mesothelioma, multivariable analysis identified age, tumour type and chemotherapy as independent prognostic factors (table 4). Sex $(p=0.44)$ was not of statistical significance. The prognostic effect of chemotherapy was modified by period and increased with time (fig. 1). For patients who did not receive chemotherapy, survival did not change over time $(p=0.23)$. For peritoneal mesothelioma, only sex (risk ratio for females, 0.61) and age were of prognostic significance in multivariable analysis (table 5). Tumour type

\begin{tabular}{|c|c|c|c|c|}
\hline TABLE 1 & $\begin{array}{l}\text { eterminar } \\
\text { atients wi }\end{array}$ & $\begin{array}{l}\text { nts of treatn } \\
\text { ith mesothe }\end{array}$ & $\begin{array}{l}\text { nent with chen } \\
\text { lioma }\end{array}$ & notherapy in \\
\hline & $\begin{array}{c}\text { Subjects } \\
n\end{array}$ & $\begin{array}{c}\text { Proportion }{ }^{\#} \\
\%\end{array}$ & $\begin{array}{c}\text { Chemotherapy } \\
\%\end{array}$ & OR $(95 \% \mathrm{Cl})$ \\
\hline \multicolumn{5}{|l|}{ Site } \\
\hline Pleural & 4464 & 94 & 17 & NS \\
\hline Peritoneal & 267 & 6 & 16 & \\
\hline \multicolumn{5}{|l|}{ Tumour type } \\
\hline Not specified & 2026 & 43 & 13 & 1 \\
\hline Sarcomatoid & 226 & 5 & 15 & $0.9(0.6-1.4)$ \\
\hline Epitheloid & 2194 & 46 & 22 & $1.4(1.1-1.6)$ \\
\hline Mixed & 285 & 6 & 15 & $1.2(0.8-1.7)$ \\
\hline \multicolumn{5}{|l|}{ Sex } \\
\hline Male & 4115 & 87 & 17 & NS \\
\hline Female & 616 & 13 & 17 & \\
\hline \multicolumn{5}{|l|}{ Age yrs } \\
\hline 20-59 & 961 & 20 & 29 & 1 \\
\hline $60-69$ & 1624 & 34 & 21 & $0.54(0.4-0.7)$ \\
\hline $70-79$ & 1630 & 35 & 11 & $0.22(0.2-0.3)$ \\
\hline$\geqslant 80$ & 516 & 11 & 3 & $0.04(0.03-0.08)$ \\
\hline \multicolumn{5}{|l|}{ Time period } \\
\hline 1995-1998 & 1377 & 29 & 8 & 1 \\
\hline 1999-2002 & 1542 & 33 & 11 & 1.4 \\
\hline 2003-2004 & 840 & 18 & 20 & 3.1 \\
\hline 2005-2006 & 972 & 21 & 36 & 8.4 \\
\hline
\end{tabular}




\begin{tabular}{lcccc} 
TABLE 2 & $\begin{array}{l}\text { Type of chemotherapy used over time (in the } \\
\text { Rotterdam region only) }\end{array}$ \\
& $\mathbf{1 9 9 5 - 1 9 9 8}$ & $\mathbf{1 9 9 9 - 2 0 0 2}$ & $\mathbf{2 0 0 3 - 2 0 0 4}$ & $\mathbf{2 0 0 5 - 2 0 0 6}$ \\
\hline Subjects n & 17 & 30 & 26 & 72 \\
Antifolate-platinum & 0 & 0 & 62 & 85 \\
Antifolate & 0 & 0 & 23 & 1 \\
Platinum & 47 & 77 & 4 & 4 \\
Other & 53 & 13 & 4 & 4 \\
Not specified & 0 & 10 & 8 & 6 \\
\hline \\
Data are presented as \%, unless otherwise stated.
\end{tabular}

$(p=0.21)$, period $(p=0.27)$ and chemotherapy $(p=0.11)$ were not of statistical significance.

\section{DISCUSSION}

This population-based series presents 4,464 patients with pleural mesothelioma with a median survival of 8.1 months, which is less than the 9.8 months reported by a large study from Italy [9]. The Italian study combined results from nine regional registries to produce a total number of 4,100 cases, diagnosed in the period 1990-2001. A recent study from

\begin{tabular}{|c|c|c|c|}
\hline \multirow[t]{2}{*}{ TABLE 3} & \multicolumn{3}{|c|}{$\begin{array}{l}\text { Determinants of survival in patients with } \\
\text { mesothelioma }\end{array}$} \\
\hline & $\begin{array}{l}\text { Median survival } \\
\text { months }\end{array}$ & $\begin{array}{l}2-y r \text { survival } \\
\%\end{array}$ & $\mathrm{p}$-value \\
\hline \multicolumn{4}{|l|}{ Site } \\
\hline Pleural & 8.1 & 13 & $<0.001$ \\
\hline Peritoneal & 3.9 & 13 & \\
\hline \multicolumn{4}{|l|}{ Tumour type } \\
\hline Not specified & 6.1 & 11 & $<0.001$ \\
\hline Sarcomatoid & 3.6 & 8 & \\
\hline Epitheloid & 10.1 & 16 & \\
\hline Mixed & 7.1 & 9 & \\
\hline \multicolumn{4}{|l|}{ Sex } \\
\hline Males & 7.7 & 13 & 0.073 \\
\hline Females & 8.1 & 15 & \\
\hline \multicolumn{4}{|l|}{ Age yrs } \\
\hline $20-59$ & 10.5 & 19 & $<0.001$ \\
\hline $60-69$ & 9.0 & 14 & \\
\hline $70-79$ & 6.5 & 10 & \\
\hline$\geqslant 80$ & 5.2 & 6 & \\
\hline \multicolumn{4}{|c|}{ No chemotherapy } \\
\hline 1995-1998 & 6.8 & 11 & 0.125 \\
\hline 1999-2002 & 7.3 & 13 & \\
\hline 2003-2004 & 6.2 & 11 & \\
\hline 2005-2006 & 7.1 & 11 & \\
\hline \multicolumn{4}{|l|}{ Chemotherapy } \\
\hline 1995-1998 & 10.1 & 16 & 0.002 \\
\hline 1999-2002 & 11.9 & 17 & \\
\hline 2003-2004 & 12.7 & 19 & \\
\hline 2005-2006 & 13.3 & 22 & \\
\hline
\end{tabular}

\section{TABLE 4 \\ Multivariable analysis of survival in patients with pleural mesothelioma}

Risk ratio $(95 \% \mathrm{Cl})$

\section{Tumour type \\ Not specified \\ Sarcomatoid \\ Epitheloid \\ Mixed \\ Age yrs \\ 20-59 \\ $60-69$ \\ $70-79$ \\ $\geqslant 80$}

Chemotherapy versus period interaction term

None

Chemotherapy 1995-1998

Chemotherapy 1999-2002

Chemotherapy 2003-2004

Chemotherapy 2005-2006
$1.33(1.15-1.53)$

$1.00(0.88-1.13)$

1

$1.16(1.06-1.26)$

$1.41(1.29-1.54)$

$1.71(1.52-1.92)$

1

$0.99(0.81-1.21)$

$0.78(0.66-0.92)$

$0.64(0.56-0.73)$
$0.76(0.71-0.81)$

$0.83(0.70-0.97)$

Western Australia [10] analysed survival trends for 1,362 patients diagnosed from 1960 to 2005 and reported improvement of median survival up to 10 months for the period 20002005. In contrast to the small series from randomised trials or specialised centres, population-based studies are not affected by selection bias, which is reflected by a high proportion of elderly patients. Median age in chemotherapy trials tends to be around $64 \mathrm{yrs}$ as frail patients get excluded for poor performance status or comorbidity.

Chemotherapy use was inversely related to age but gradually increased in time from $8 \%$ in the first period to $36 \%$ in the last period. In a population-based study from Leeds, UK [5], comprising patients diagnosed from 2002 to 2005, 37\% were considered fit for chemotherapy but one-half of those declined. Palliative chemotherapy is now considered standard treatment in Europe [11, 12], even though a recent British trial [13] comparing best supportive care, a platinum combination (mitomycin-vinblastine-cisplatin) and vinorelbin, could not demonstrate a benefit with respect to survival or quality of life for the chemotherapy arms. Despite struggles with reimbursement, the antifolate-platinum regimen was quickly adopted as a standard in the Netherlands. The actual chemotherapy use may have been higher than recorded by the cancer registry, especially when treatment was delayed until progression. In several other countries, availability was limited due to financial restrictions [14].

Median survival improved with time from 7.1 to 9.2 months, presumably related to the introduction of the new chemotherapy regimen. For the first period, prognosis seemed better after chemotherapy (10.1 versus 6.8 months), but the survival difference faded after controlling for age and tumour type. This finding confirms the 1998 review on chemotherapy trials, which concluded that none of the available agents showed meaningful efficacy [1]. The chemotherapy-period interaction term suggests that chemotherapy became more effective with 


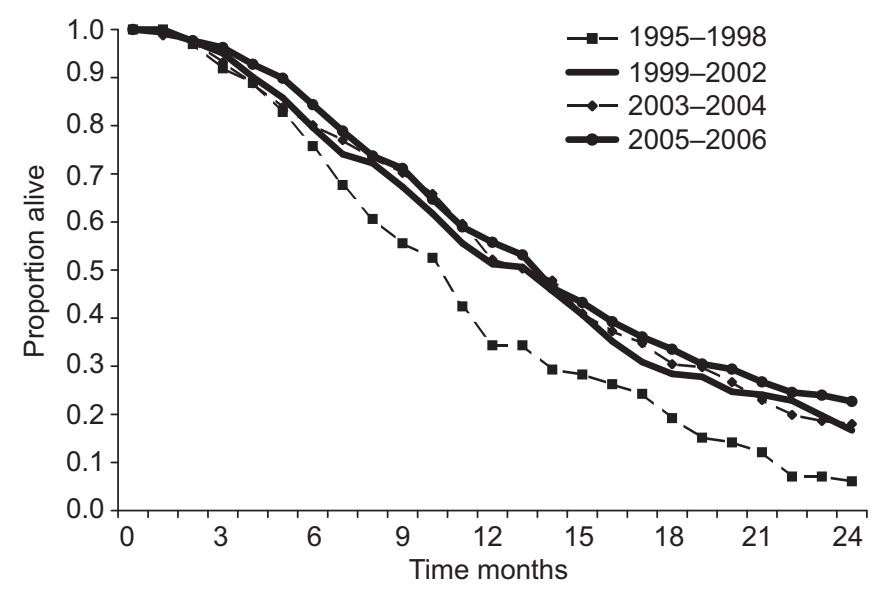

FIGURE 1. 2-yr survival for patients with malignant pleural mesothelioma who were treated with chemotherapy, stratified by period of diagnosis.

time but efficacy of chemotherapy cannot be reliably evaluated in observational studies. Lack of information on important prognostic factors, such as extent of disease or performance status, may introduce residual confounding. Even with modern methodology, such as propensity scores and instrumental variable methods [15], selection bias cannot be ruled out. Immortal time bias [16] poses another problem in nonrandomised studies as survival is calculated from day of diagnosis and, by definition, chemotherapy patients cannot die between diagnosis and their first cycle, hence getting a survival head start. The latter bias may also explain why survival appears to be better for patients who receive post-study chemotherapy [17].

Other than more and better chemotherapy, several alternative explanations may clarify the increase in survival. For example, expertise in palliative care has certainly increased over the past decade. Also, tumours may be diagnosed earlier [10] due to wider knowledge of the effects of asbestos exposure, a less pessimistic view on the clinical management of mesothelioma patients or to better prospects for financial compensation after confirmation of the diagnosis [18].

Multivariable survival analysis also identified age and histotype as independent prognostic factors for pleural mesothelioma. At a greater age, patients are less able to cope with a

\begin{tabular}{lc} 
TABLE 5 & $\begin{array}{l}\text { Multivariable analysis of survival in patients with } \\
\text { peritoneal mesothelioma }\end{array}$ \\
Risk ratio $\mathbf{( 9 5 \%} \mathbf{~ C l )}$ \\
\hline Sex & 1 \\
Males & $0.61(0.45-0.84)$ \\
Females & 1 \\
Age yrs & $1.86(1.27-2.73)$ \\
$20-59$ & $2.53(1.69-3.79)$ \\
$60-69$ & $2.34(1.36-4.03)$ \\
$70-79$ &
\end{tabular}

disease that is characterised by local tumour progression. In other types of cancer, survival is often determined by progression of distant metastases but, in pleural mesothelioma, the symptom burden is inflicted by invasion of intrathoracic structures [11]. The prognostic impact of age may, however, be confounded by differences in stage distribution. Diagnostic delay tends to be longer for the elderly, which will produce a higher stage at diagnosis and hence a poorer survival. A staging system for mesothelioma has been developed [19], but current imaging techniques have limited value in predicting local tumour spread or involvement of mediastinal lymph nodes [20]. Surgical staging provides more accurate information, but this is only performed when patients are considered eligible for radical surgery. Stage bias may even explain the poor prognosis for sarcomatoid tumours, known for their more invasive growth pattern.

The clinical behaviour of peritoneal mesothelioma is clearly different from its pleural counterpart. Survival was much poorer, probably due to later diagnosis. Similar to the situation for ovarian cancer, symptoms tend to be nonspecific and tumour load can be extensive at diagnosis. Females comprise $23 \%$ of peritoneal mesothelioma cases against $12 \%$ of pleural mesothelioma. The prolonged survival for females may be related to earlier diagnosis or to misclassification of ovarian tumours. Our median survival of 3.9 months is evidently lower than the 5.6 months reported for Italy [21], but difficult to compare because $41 \%$ of cases were female in the Italian study. Response rates to antifolate-platinum treatment are similar to those for pleural lesions [22]. Cytoreductive surgery followed by hyperthermic intraperitoneal chemotherapy might be a promising treatment alternative given the $47 \%$ 5-yr survival reported by YAN et al. [23], albeit in selected patients with a mean age of 50 yrs.

In conclusion, despite introduction of novel chemotherapy regimens, MM is still a fatal disease with a median prognosis of $<1 \mathrm{yr}$. With disease incidence at its peak, now is the time to test new treatment options, preferably in randomised controlled trials. Within $10 \mathrm{yrs}$, the number of mesothelioma patients will decrease and the ageing of the patient population will make them less suitable for inclusion in trials with toxic treatment. Quality of life should be a major end-point in these trials and prevention remains the best option to decrease mortality from mesothelioma.

\section{SUPPORT STATEMENT}

The present study was supported by a Research Grant from the Institute for Asbestos Victims.

\section{STATEMENT OF INTEREST}

None declared.

\section{REFERENCES}

1 Ryan CW, Herndon J, Vogelzang NJ. A review of chemotherapy trials for malignant mesothelioma. Chest 1998; 113: Suppl. 1, 66S-73S.

2 Hughes A, Calvert P, Azzabi A, et al. Phase I clinical and pharmacokinetic study of pemetrexed and carboplatin in patients with malignant pleural mesothelioma. J Clin Oncol 2002; 20: 3533-3544.

3 Vogelzang NJ, Rusthoven JJ, Symanowski J, et al. Phase III study of pemetrexed in combination with cisplatin versus cisplatin alone in 
patients with malignant pleural mesothelioma. J Clin Oncol 2003; 21: 2636-2644.

4 van Meerbeeck JP, Gaafar R, Manegold C, et al. Randomized phase III study of cisplatin with or without raltitrexed in patients with malignant pleural mesothelioma: an intergroup study of the European Organisation for Research and Treatment of Cancer Lung Cancer Group and the National Cancer Institute of Canada. J Clin Oncol 2005; 23: 6881-6889.

5 Chapman A, Mulrennan S, Ladd B, et al. Population based epidemiology and prognosis of mesothelioma in Leeds, UK. Thorax 2008; 63: 435-439.

6 van Sandick JW, Kappers I, Baas P, et al. Surgical treatment in the management of malignant pleural mesothelioma: a single institution's experience. Ann Surg Oncol 2008; 15: 1757-1764.

7 Santoro A, O'Brien ME, Stahel RA, et al. Pemetrexed plus cisplatin or pemetrexed plus carboplatin for chemonaïve patients with malignant pleural mesothelioma: results of the International Expanded Access Program. J Thorac Oncol 2008; 3: 756-763.

8 Baas P, van Klaveren RJ, Kraaij K. Treatment of malignant pleural mesothelioma patients with pemetrexed: subanalyses of the Dutch expanded access program. Ned Tijdschr Oncol 2009; 6: 292-301.

9 Montanaro F, Rosato R, Gangemi M, et al. Survival of pleural malignant mesothelioma in Italy: a population-based study. Int J Cancer 2009; 124: 201-207.

10 Musk AW, Olsen N, Alfonso $\mathrm{H}$, et al. Predicting survival for malignant mesothelioma. Eur Respir J 2011; 38: 1420-1424.

11 van Meerbeeck JP, Scherpereel A, Surmont VF, et al. Malignant pleural mesothelioma: the standard of care and challenges for future management. Crit Rev Oncol Hematol 2011; 78: 92-111.

12 Scherpereel A, Astoul P, Baas P, et al. Guidelines of the European Respiratory Society and the European Society of Thoracic Surgeons for the management of malignant pleural mesothelioma. Eur Respir J 2010; 35: 479-495.

13 Muers MF, Stephens RJ, Fisher P, et al. Active symptom control with or without chemotherapy in the treatment of patients with malignant pleural mesothelioma (MS01): a multicentre randomised trial. Lancet 2008; 371: 1685-1694.

14 Burkitt V. In Australia, patients and government at odds over mesothelioma treatment costs. J Natl Cancer Inst 2007; 99: 1750-1752.

15 Bosco JL, Silliman RA, Thwin SS, et al. A most stubborn bias: no adjustment method fully resolves confounding by indication in observational studies. J Clin Epidemiol 2010; 63: 64-74.

16 Suissa S. Effectiveness of inhaled corticosteroids in chronic obstructive pulmonary disease: immortal time bias in observational studies. Am J Respir Crit Care Med 2003; 168: 49-53.

17 Manegold C, Symanowski J, Gatzemeier U, et al. Second-line (poststudy) chemotherapy received by patients treated in the phase III trial of pemetrexed plus cisplatin versus cisplatin alone in malignant pleural mesothelioma. Ann Oncol 2005; 16: 923-927.

18 Baas $\mathrm{P}$, van 't Hullenaar N, Wagenaar J, et al. Occupational asbestos exposure: how to deal with suspected mesothelioma cases-the Dutch approach. Ann Oncol 2006; 17: 848-852.

19 Rusch VW. A proposed new international TNM staging system for malignant pleural mesothelioma. From the International Mesothelioma Interest Group. Chest 1995; 108: 1122-1128.

20 Heelan RT, Rusch VW, Begg CB, et al. Staging of malignant pleural mesothelioma: comparison of $\mathrm{CT}$ and MR imaging. AJR Am J Roentgenol 1999; 172: 1039-1047.

21 Mirabelli D, Roberti S, Gangemi M, et al. Survival of peritoneal malignant mesothelioma in Italy: a population-based study. Int $J$ Cancer 2009; 124: 194-200.

22 Carteni G, Manegold C, Garcia GM, et al. Malignant peritoneal mesothelioma - results from the International Expanded Access Program using pemetrexed alone or in combination with a platinum agent. Lung Cancer 2009; 64: 211-218.

23 Yan TD, Deraco M, Baratti D, et al. Cytoreductive surgery and hyperthermic intraperitoneal chemotherapy for malignant peritoneal mesothelioma: multi-institutional experience. J Clin Oncol 2009; 27: 6237-6242. 